\title{
On Some Inequalities Involving Harmonic Mean and Moments
}

\author{
MADHU GUPTA ${ }^{1, *}$, RAJESH SHARMA ${ }^{2}$ AND S.R. SHARMA ${ }^{3}$ \\ ${ }^{1}$ Department of Mathematics, Chitkara University, Solan, India \\ ${ }^{2}$ Department of Mathematics, H.P.U., Shimla, India \\ ${ }^{3}$ Department of Mathematics, Chitkara University, Solan, India
}

Email: madhu.gupta@chitkarauniversity.edu.in

Received: July 22, 2017 I Revised: August 25, 2017 | Accepted: August 30, 2017

Published Online: September 01, 2017

The Author(s) 2017. This article is published with open access at www.chitkara.edu.in/publications

\begin{abstract}
Abstact: We derive bounds on the second order moment of a random variable in terms of its arithmetic and harmonic means. Both discrete and continuous cases are considered and it is shown that the present bounds provide refinements of the bounds which exist in literature. As an application we obtain a lower bound for the spread of a positive definite matrix $A$ in terms of traces of $A, A^{-1}$ and $A^{2}$. Our results compare favourably with those obtained by Wolkowicz and Styan (Bounds for eigenvalues using traces, Lin. Alg. Appl. 29, 471-506, 1980).
\end{abstract}

Keywords: Harmonic mean, variance, positive definite matrix, spread.

\section{INTRODUCTION}

Let a random variable $x$, discrete or continuous takes values in the interval a $\leq x \leq b$. Let $H, \mu_{1}^{\prime}$ and $\mu_{2}^{\prime}$ respectively denote the harmonic mean, arithmetic mean and second order moment about origin of the random variable $x$. The well-known Kantorovich inequality says that [1]

$$
\frac{\mu_{1}^{\prime}}{H} \leq \frac{(a+b)^{2}}{4 a b}, a>0
$$

The inequality (1.1) follows from the inequality [2],

$$
\mu_{1}^{\prime} \leq a+b-\frac{a b}{H}
$$

Mathematical Journal of Interdisciplinary Sciences Vol-6, No-1, September 2017 pp. 54-65 
Gupta, M

Sharma, R

Sharma, SR

Sharma [3] shows that

$$
\frac{\mu_{1}^{\prime}}{H} \leq \frac{(a+S)^{2}}{a(a+2 S)} \leq \frac{(a+b)^{2}}{4 a b}, a>0 .
$$

where $S^{2}$ is the variance of the random variable $x$. The inequality (1.3) provides a refinement of (1.1). Several authors have worked on such inequalities, their further refinements, extensions and applications. In particular, Krasnosel'skii and Krein [4] proved that

$$
\frac{\mu_{2}^{\prime}}{\mu_{1}^{\prime 2}} \leq \frac{(a+b)^{2}}{4 a b}, a>0 .
$$

The inequality (1.4) follows from the inequality [2],

$$
\mu_{2}^{\prime} \leq(a+b) \mu_{1}^{\prime}-a b
$$

We note that the inequalities (1.1) and (1.2) involve $\mu_{1}^{\prime}$ and $H$ while (1.4) and (1.5) involve $\mu_{1}^{\prime}$ and $\mu_{2}^{\prime}$. Our main result (Theorem-2.1 and 2.2, below) relate $\mu_{1}^{\prime}, \mu_{2}^{\prime}$ and $H$ at the same time, and provides refinements of the inequalities (1.5), (Corollary-2.1, below). The bounds for the harmonic mean are deduced (Corollary-2.2, below). A refinement of the Krasnosel'skii and Krein inequality (1.4) is also obtained, (Corollary-2.3, below). As an application we obtain a lower bound for the spread of a positive definite matrix $A$ (Theorem-3.1, below) in terms of traces of $A, A^{-1}$ and $A^{2}$.

\section{MAIN RESULTS}

\section{Theorem-2.1}

Let a discrete random variable takes a finite set of real values $x_{1}, x_{2}, \ldots, x_{n}$ with corresponding probabilities $p_{1}, p_{2}, \ldots, p_{n}$. Let $0<a \leq x_{i} \leq b, i=1,2, \ldots, n$ ' and $a<H<b$. Then

$$
\mu_{2}^{\prime} \leq b \mu_{1}^{\prime}-\frac{\left(b-\mu_{1}^{\prime}\right)^{2} H}{b-H}
$$

and

$$
\mu_{2}^{\prime} \geq a \mu_{1}^{\prime}+\frac{\left(\mu_{1}^{\prime}-a\right)^{2} H}{H-a}
$$


The inequalities (2.1) and (2.2) become equalities when $n=2$.

\section{Proof}

For $0<a \leq x_{i} \leq b$, we have $\left(x_{i}-\alpha\right)^{2}\left(x_{i}-b\right) \leq 0$ for any real number $\alpha, i=1,2, \ldots, n$. This gives

$$
x_{i}^{2}-(2 \alpha+b) x_{i}+\alpha(\alpha+2 b)-\frac{\alpha^{2} b}{x_{i}} \leq 0,
$$

$i=1,2, \ldots, n$. Multiplying both sides of $(2.3)$ by $p_{\mathrm{i}}$ and adding these $\mathrm{n}$ inequalities, we get on simplification,

$$
(b-H) \alpha^{2}-2 H\left(b-\mu_{1}^{\prime}\right) \alpha+H\left(b \mu_{1}^{\prime}-\mu_{2}^{\prime}\right) \geq 0 .
$$

The inequality (2.4) holds for all real values of $\alpha$ and therefore its discriminant must be non positive. Hence, we must have

$$
4 H^{2}\left(b-\mu_{1}^{\prime}\right)^{2}-4 H(b-H)\left(b \mu_{1}^{\prime}-\mu_{2}^{\prime}\right) \leq 0 .
$$

The inequality (2.1) now follows easily from (2.5). On using similar arguments we find that

$$
(H-a) \beta^{2}-2 H\left(\mu_{1}^{\prime}-a\right) \beta+H\left(\mu_{2}^{\prime}-a \mu_{1}^{\prime}\right) \geq 0 .
$$

The inequality (2.2) now follows from (2.6); the discriminant of the quadratic equation in (2.6) must be non positive. Consider the system of linear equations

$$
\begin{gathered}
p_{1}+p_{2}+p_{3}=1, \\
x_{1} p_{1}+x_{2} p_{2}+x_{3} p_{3}=\mu_{1}^{\prime}
\end{gathered}
$$

and

$$
x_{1}^{2} p_{1}+x_{2}^{2} p_{2}+x_{3}^{2} p_{3}=\mu_{2}^{\prime} .
$$


Gupta, M

Sharma, R

Sharma, SR

From (2.7), (2.8) and (2.9), we have

$$
\begin{gathered}
p_{1}=\frac{\mu_{2}^{\prime}-\left(x_{2}+b\right) \mu_{1}^{\prime}+x_{2} b}{\left(a-x_{2}\right)(a-b)}, \\
p_{2}=\frac{\mu_{2}^{\prime}-(a+b) \mu_{1}^{\prime}+a b}{\left(x_{2}-a\right)\left(x_{2}-b\right)}
\end{gathered}
$$

and

$$
p_{3}=\frac{\mu_{2}^{\prime}-\left(a+x_{2}\right) \mu_{1}^{\prime}+a x_{2}}{(b-a)\left(b-x_{2}\right)} .
$$

On substituting the values of $p_{1}, p_{2}$ and $p_{3}$ respectively from (2.10), (2.11) and (2.12) in

$$
\frac{1}{x_{1}} p_{1}+\frac{1}{x_{2}} p_{2}+\frac{1}{x_{3}} p_{3}=\frac{1}{H},
$$

we get the following cubic equation in variable $x_{2}$,

$$
\left(x_{2}-a\right)\left(x_{2}-b\right)\left(x_{2}-\frac{\mu_{2}^{\prime}-(a+b) \mu_{1}^{\prime}+a b}{\mu_{1}^{\prime}-(a+b)+\frac{a b}{H}}\right)=0 .
$$

It is easily seen that the root

$$
x_{2}=\frac{\mu_{2}^{\prime}-(a+b) \mu_{1}^{\prime}+a b}{\mu_{1}^{\prime}-(a+b)+\frac{a b}{H}}
$$

of cubic equation (2.14) lies in the interval $[a, b]$. If equality sign holds in (2.1) then on substituting

$$
H=\frac{b\left(b \mu_{1}^{\prime}-\mu_{2}^{\prime}\right)}{\left(b-\mu_{1}^{\prime}\right)^{2}+\left(b \mu_{1}^{\prime}-\mu_{2}^{\prime}\right)}
$$


in (2.15) we find that

$$
x_{2}=\frac{b \mu_{1}^{\prime}-\mu_{2}^{\prime}}{b-\mu_{1}^{\prime}} .
$$

From (2.10)-(2.12) and (2.17),we get that $p_{1}=0$,

$$
p_{2}=\frac{\left(b-\mu_{1}^{\prime}\right)^{2}}{\mu_{2}^{\prime}-\mu_{1}^{\prime 2}+\left(b-\mu_{1}^{\prime}\right)^{2}}
$$

and

$$
p_{2}=\frac{\mu_{2}^{\prime}-\mu_{1}^{\prime 2}}{\mu_{2}^{\prime}-\mu_{1}^{\prime 2}+\left(b-\mu_{1}^{\prime}\right)^{2}} .
$$

Thus if equality sign holds in (2.1) then only two of the probabilities are nonzero, $\mathrm{n}=2$. Similarly, we can show that if equality sign holds in (2.2) then $\mathrm{n}$ $=2$. Conversly, if $\mathrm{n}=2$, we must have

$$
\mu_{2}^{\prime}=(a+b) \mu_{1}^{\prime}-a b .
$$

From (2.10)-(2.12) and (2.20),we see that $p_{2}=0$,

$$
p_{1}=\frac{b-\mu_{1}^{\prime}}{b-a} \text { and } p_{3}=\frac{\mu_{1}^{\prime}-a}{b-a} \text {. }
$$

Therefore

$$
\begin{aligned}
a^{2} p_{1}+b^{2} p_{2} & =a^{2} \frac{b-\mu_{1}^{\prime}}{b-a}+b^{2} \frac{\mu_{1}^{\prime}-a}{b-a} \\
& =b \mu_{1}^{\prime}-\frac{H\left(b-\mu_{1}^{\prime}\right)^{2}}{b-H} \\
& =a \mu_{1}^{\prime}+\frac{H\left(\mu_{1}^{\prime}-a\right)^{2}}{H-a} .
\end{aligned}
$$

Hence, the inequalities (2.1) and (2.2) reduce to equalities when $n=2$. 
Gupta, M

Sharma, R

Sharma, SR

\section{Theorem-2.2}

The inequalities (2.1) and (2.2) also hold good when $H, \mu_{1}^{\prime}$ and $\mu_{2}^{\prime}$ are respectively harmonic mean, arithmetic mean and second order moment about origin of a continuous random variable $x$ whose probability density function $\phi(x)$ takes non-zero values in the interval $[\mathrm{a}, \mathrm{b}]$, such that $\mathrm{a}<\mathrm{H}<\mathrm{b}$, and $\mathrm{a}>0$.

\section{Proof:}

For $0<a \leq x \leq b$, we have $(x-\alpha)^{2}(x-b) \leq 0$ for any real number $\alpha$.This gives

$$
x^{2}-(2 \alpha+b) x+\alpha(\alpha+2 b)-\frac{\alpha^{2} b}{x} \leq 0 .
$$

Multiplying both sides the inequality (2.22) by probability density function $\phi(x)$, we get on using the properties of the definite integral,

$$
(b-H) \alpha^{2}-2\left(b-\mu_{1}^{\prime}\right) H \alpha+\left(b \mu_{1}^{\prime}-\mu_{2}^{\prime}\right) H \geq 0 .
$$

This shows that inequality (2.1) remains valid when $x$ is a continuous random variable. On using similar arguments we can show that (2.2) also hold good for the case when $x$ is a continuous random variable.

\section{Corollary-2.1}

For a random variable which is discrete or continuous and takes values in the interval $[a, b]$, we have

$$
\mu_{2}^{\prime} \leq(a+b) \mu_{1}^{\prime}-a b-\frac{H\left(b-\mu_{1}^{\prime}\right)}{b-H}\left(a+b-\frac{a b}{H}-\mu_{1}^{\prime}\right) .
$$

The inequality (2.24) provides the refinement of the inequality (1.5).

\section{Proof:}

From the inequality (2.1), we have,

$$
\mu_{2}^{\prime} \leq(a+b) \mu_{1}^{\prime}-a b-\frac{H\left(b-\mu_{1}^{\prime}\right)}{b-H}\left(a+b-\frac{a b}{H}-\mu_{1}^{\prime}\right) .
$$

Also 


$$
\begin{aligned}
& \frac{\mu_{1}^{\prime 2}(b-H)+b\left(b-\mu_{1}^{\prime}\right)\left(\mu_{1}^{\prime}-H\right)}{b-H} \\
& \times\left(a+b-\frac{a b}{H}-\mu_{1}^{\prime}\right) \\
& =(a+b) \mu_{1}^{\prime}-a b-\frac{H\left(b-\mu_{1}^{\prime}\right)}{b-H} \\
& \times\left(a+b-\frac{a b}{H}-\mu_{1}^{\prime}\right) .
\end{aligned}
$$

Combining (2.25) and (2.26) we immediately get (2.24).

\section{Corollary-2.2}

For a discrete or continuous random variable varying over the interval [a,b], we have

$$
H \leq \frac{b\left(b \mu_{1}^{\prime}-\mu_{2}^{\prime}\right)}{\left(b-\mu_{1}^{\prime}\right)^{2}+b \mu_{1}^{\prime}-\mu_{2}^{\prime}}
$$

and

$$
H \geq \frac{a\left(\mu_{2}^{\prime}-a \mu_{1}^{\prime}\right)}{\mu_{2}^{\prime}-a \mu_{1}^{\prime}-\left(\mu_{1}^{\prime}-a\right)^{2}} .
$$

\section{Proof:}

The inequalities (2.27) and (2.28) follow easily from (2.1) and (2.2), respectively.

\section{Corollary-2.3}

For a random variable which is discrete or continuous and takes values in the interval $[a, b]$, we must have

$$
\frac{\mu_{2}^{\prime}}{\mu_{1}^{\prime 2}} \leq \frac{(a+b)^{2}}{4 a b}-\frac{\left[\left(a^{2}+b^{2}\right) H-(a+b) a b\right]^{2}}{4 a b H\left[\left(a^{2}+a b+b^{2}\right) H-(a+b) a b\right]} .
$$

The inequality (2.29) provides a refinement of the Krasnosel'skii and Krein inequality (1.4). 
Gupta, M

Sharma, R

Sharma, SR

\section{Proof:}

We note that

$$
\frac{\mu_{2}^{\prime}}{\mu_{1}^{\prime 2}} \leq \frac{\sigma^{2}}{\mu_{1}^{\prime 2}}+1
$$

Therefore it suffices to prove that

$$
\frac{\sigma^{2}}{\mu_{1}^{\prime 2}} \leq \frac{(b-a)^{2}}{4 a b}-\frac{\left[\left(a^{2}+b^{2}\right) H-(a+b) a b\right]^{2}}{4 a b H\left[\left(a^{2}+a b+b^{2}\right) H-(a+b) a b\right]}
$$

Multiplying both sides of the inequalities (2.1) and (2.2) respectively by b and (-a) we get on adding the resulting inequalities:

$$
\begin{aligned}
& \frac{\sigma^{2}}{\mu_{1}^{\prime 2}} \leq \frac{\left(\mu_{1}^{\prime}-H\right)}{\mu_{1}^{\prime 2}(H-a)(b-H)} \\
& \quad \times\left[\left(a^{2}+a b+b^{2}\right) H-(a+b) a b-\mu_{1}^{\prime}((a+b) H-a b)\right] .
\end{aligned}
$$

Consider a function

$$
f\left(\mu_{1}^{\prime}\right) \leq \frac{\left(\mu_{1}^{\prime}-H\right)}{\mu_{1}^{\prime 2}}\left[x-\mu_{1}^{\prime} y\right]
$$

where

$$
x=\left(a^{2}+a b+b^{2}\right) H-(a+b) a b
$$

and

$$
y=(a+b) H-a b .
$$

The derivative

$$
f^{\prime}\left(\mu_{1}^{\prime}\right) \leq \frac{2 H x-(x+H y) \mu_{1}^{\prime}}{\mu_{1}^{\prime 3}}
$$

vanishes at 


$$
\mu_{1}^{\prime}=\frac{2 H x}{x+H y} .
$$

The function $f\left(\mu_{1}^{\prime}\right)$ has maximum at the value of $\mu_{1}^{\prime}$ given by (2.37). Hence

$$
f\left(\mu_{1}^{\prime}\right) \leq \frac{(a+b)^{2}(H-a)(b-H)}{4 H x} .
$$

Combining (2.33), (2.34), (2.35) and (2.38), we easily get the inequality (2.31). It is evident that the inequality (2.29) provides a refinement of the inequality (1.4). We also note here that the function

$$
g(H) \leq \frac{(a+b)^{2}(H-a)(b-H)}{4\left(\left(a^{2}+a b+b^{2}\right) H^{2}-(a+b) a b H\right)} .
$$

The derivative

$$
\begin{aligned}
& g^{\prime}(H) \\
& \leq \frac{(a+b)^{2}}{4} \frac{2 a b\left(a^{2}+a b+b^{2}\right)-\left(a^{2}+b^{2}\right)(a+b) H^{2}-(a+b) a^{2} b^{2}}{\left(\left(a^{2}+a b+b^{2}\right) H^{2}-(a+b) a b H\right)^{2}}
\end{aligned}
$$

has maximum at

$$
H=\frac{(a+b) a b}{a^{2}+b^{2}} .
$$

Therefore

$$
g(H) \leq \frac{(b-a)^{2}}{4 a b}
$$

\section{AN APPLICATION}

Let $\lambda_{1}, \lambda_{2}, \ldots, \lambda_{n}$, be the eigenvalues of an $\mathrm{n} \times \mathrm{n}$ matrix $\mathrm{A}$. The spread of $\mathrm{A}$ is defined by

$$
s(A)=\max _{i, j}\left|\lambda_{i}-\lambda_{j}\right| .
$$


Gupta, M

Sharma, R

Sharma, SR

This quantity of Hermition and positive definite matrices is important in matrix analysis, and has applications in combinatorial optimization problems [5]. Several authors have given bounds for the spread. In particular, Wolkowicz and Styan [6], have obtained the following lower bound for the spread of positive definite matrix in terms of traces of $\mathrm{A}$ and $\mathrm{A}^{2}$,

$$
s(A) \geq 2 \sqrt{\frac{\operatorname{tr} A^{2}}{n}-\left(\frac{\operatorname{tr} A}{n}\right)^{2}}, \text { neven }
$$

and

$$
s(A) \geq \frac{2 n}{\sqrt{n^{2}-1}} \sqrt{\frac{\operatorname{tr} A^{2}}{n}-\left(\frac{\operatorname{tr} A}{n}\right)^{2}}, \mathrm{n} \geq 1 \text { odd }
$$

Here we obtain a lower bound for the spread of positive definite matrix in terms of traces of $A, A^{-1}$ and $A^{2}$. Our results compare favourably then those obtained by Wolkowicz and Styan [6], (Example 1 and 2, below).

\section{Theorem-3.1}

Let $\mathrm{A}$ be $\mathrm{n} \times \mathrm{n}$ positive definite matrix with eigenvalues $0<\lambda_{1} \leq \lambda_{2} \leq \ldots \leq \lambda_{n}$. Then

$$
s(A) \geq \frac{\sqrt{\left(\operatorname{tr} A^{2} \operatorname{tr} A^{-1}-n \operatorname{tr} A\right)^{2}-4\left(\operatorname{tr} A \operatorname{tr} A^{-1}-n^{2}\right)\left(n \operatorname{tr} A^{2}-(\operatorname{tr} A)^{2}\right)}}{\operatorname{tr} A \operatorname{tr} A^{-1}-n^{2}}
$$

\section{Proof:}

Let $H, \mu_{1}^{\prime}$ and $\mu_{2}^{\prime}$ respectively denote the harmonic mean, arithmetic mean and second order moment about origin of the $\mathrm{n}$ eigenvalues $\lambda_{1}, \lambda_{2}, \ldots, \lambda_{n}$. Then

$$
\begin{aligned}
& \mu_{1}^{\prime}=\frac{\operatorname{trA}}{n}, \\
& \mu_{2}^{\prime}=\frac{t r A^{2}}{n}
\end{aligned}
$$

and 


$$
H=\frac{n}{\operatorname{tr} A^{-1}} .
$$

From the inequalities (2.1) and (2.2) we respectively find that

$$
\lambda_{n} \geq \frac{\mu_{2}^{\prime}-H \mu_{1}^{\prime}+\sqrt{\left(\mu_{2}^{\prime}-H \mu_{1}^{\prime}\right)^{2}-4\left(\mu_{1}^{\prime}-H\right)\left(\mu_{2}^{\prime}-\mu_{1}^{\prime 2}\right) H}}{2\left(\mu_{1}^{\prime}-H\right)}
$$

and

$$
\lambda_{1} \leq \frac{\mu_{2}^{\prime}-H \mu_{1}^{\prime}-\sqrt{\left(\mu_{2}^{\prime}-H \mu_{1}^{\prime}\right)^{2}-4\left(\mu_{1}^{\prime}-H\right)\left(\mu_{2}^{\prime}-\mu_{1}^{\prime 2}\right) H}}{2\left(\mu_{1}^{\prime}-H\right)}
$$

On subtracting (3.9) from (3.8), we get that

$$
\lambda_{n}-\lambda_{1} \geq \frac{\sqrt{\left(\mu_{2}^{\prime}-H \mu_{1}^{\prime}\right)^{2}-4\left(\mu_{1}^{\prime}-H\right)\left(\mu_{2}^{\prime}-\mu_{1}^{\prime 2}\right) H}}{\mu_{1}^{\prime}-H}
$$

On substituting values of $\mu_{1}^{\prime}, \mu_{2}^{\prime}$ and $H$ respectively from equations (3.5), (3.6) and (3.7) in the inequality (3.10), we immediately get (3.4).

\section{Example 1.}

Let

$$
A=\left|\begin{array}{llll}
4 & 0 & 2 & 3 \\
0 & 5 & 0 & 1 \\
2 & 0 & 6 & 0 \\
3 & 1 & 0 & 7
\end{array}\right|
$$

We have, $\operatorname{tr} A=22, \operatorname{tr} A^{2}=154$ and $\operatorname{tr} A^{-1}=\frac{481}{410}$. From the inequality we get $s(A) \geq 5.950$, where as Wolkowicz and Styan [6] have shown than $s(A) \geq 5.744$. 
Gupta, M

Sharma, R

Sharma, SR

\section{Example 2.}

Let

$$
A=\left|\begin{array}{llll}
1 & 1 & 1 & 1 \\
1 & 2 & 0 & 1 \\
1 & 0 & 3 & 1 \\
1 & 1 & 1 & 4
\end{array}\right|
$$

We have $\operatorname{tr} A=10, \operatorname{tr} A^{2}=40$ and $\operatorname{tr} A^{-1}=\frac{29}{3}$. From the inequality we get $s(A) \geq 3.9362$, where as Wolkowicz and Styan [6] have shown that $s(A) \geq 3.8730$.

\section{REFERENCES}

[1] Kantorovich, L.V., Functional analysis and applied mathematics [in Russian], Uspekki Matematicheskikh Nauk 3, 89-185, (1948).

[2] Goldman, A. J., A generalization of Rennie's inequality J. Res. Nat. Bur. Standards Sect. B, 68 B, 59-63 (1964).

[3] Sharma, R., Some more inequalities for arithmetic mean, harmonic mean and variance, Math. Inequal. Appl., 2, 109-114, (2008).

[4] Krasnosel'skii, M. A. and Kreïn, S. G., An iteration process with minimal residuals [in Russian], Mat. Sbornik N.S. 31 (73), 315-334, (1952).

[5] Merikoski, J.K., On the Trace and Sum of elements of a Matrix, Linear Algebra and Its Applications, 60: 177-185, (1984).

[6] Wolkowicz, H. and Styan, P.H., Bounds for Eigenvalues Using Traces, Linear Algebra and Its Applications, 29: 471-506, (1980). 